\title{
Über die mittlere Parallaxe der Sterne 10. Größe.
}

In den Jahren r907-09 habe ich die Engelhardtschen Begleiter der Bradley-Sterne, in der Absicht, einen Beitrag zur Ermittelung ihrer Eigenbewegungen zu liefern, am IoZöller der Urania-Sternwarte gemessen. Obgleich das Zeitintervall im Mittel nur 19.0 Jahre beträgt, habe ich doch geglaubt, die Messungen zu einer vorläufigen Prüfung der Comstockschen Resultate ${ }^{1}$ ) benutzen zu sollen.

Die Engelhardtschen Bradley-Sterne dürften in der Tat bei künftigen Untersuchungen über die Eigenbewegungen der lichtschwachen Sterne von der größten Bedeutung werden. Die Eigenbewegungen der Hauptsterne sind mit großer Genauigkeit bestimmt; nach Prof. Boss betragen die w. F. der Eigenbewegungen im Mittel nur o." 2 im Jahrhundert. Ferner wurden in das Engelhardtsche Verzeichnis nur Sterne mit Eigenbewegungen über $\circ$." I jährlich aufgenommen; ein endgültiges Urteil über den Charakter der relativen Bewegungen dürfte daher schon nach wenigen Jahrzehnten möglich sein. Die Engelhardtschen Sterne sind weiter sämtlich von einem Beobachter gemessen worden, wodurch die Elimination der konstanten Messungsfehler erheblich erleichtert wird. Es dürfte schließlich noch ein großer Vorteil dieser Sterne sein, daß bei der Auswahl auf die Größe der relativen Bewegungen keine Rücksicht genommen wurde, weshalb man zu der Annahme berechtigt ist, daß die Engelhardtschen Sterne wirklich ein typisches Bild der Verhältnisse der Sterne $1 \mathrm{o}^{\mathrm{mn}}$ liefern können.

Die Messungen dieser lichtschwachen Sterne waren am Urania-Refraktor, namentlich in den hellen Sommernächten, recht schwierig. Bei ruhigen Bildern ist die Luft hier meistens sichtbar. Bei sehr reiner Luft sind die Bilder dagegen fast immer wallend, und die Hauptsterne erscheinen dann häufig als explodierende Lichtmassen. Ob die Luftverhältnisse in Bemerkungen im èrsten Teile seiner Beobachtungen ${ }^{2}$ ) sehr fraglich. Die w. F. meiner Messungen, die mit roter Feldbeleuchtung ausgeführt werden mußten, zeigen ein rascheres Anwachsen mit abnehmender Helligkeit als die Engelhardtschen, wie aus dem folgenden Täfelchen ersichtlich ist:

\begin{tabular}{|c|c|c|c|c|}
\hline Helligkeit & \multicolumn{2}{|c|}{ v. Engelhardt } & \multicolumn{2}{|c|}{ Lau } \\
\hline des Begleiters & $s \cdot \mathrm{d} p$ & d.s & $s \cdot \mathrm{d} p$ & $\mathrm{~d} s$ \\
\hline $8^{m} \cdot 5^{-} 9^{m} \cdot 5$ & $\pm 0 . "$ I 5 & $\pm 0 . "$ I 3 & \pm I." โ 4 & \pm 0. I 4 \\
\hline $9.5-10.5$ & $\pm 0.2 \mathrm{I}$ & \pm 0.11 & \pm 0.20 & \pm 0.20 \\
\hline I0.5- I I. 5 & \pm 0.25 & \pm 0.15 & \pm 0.34 & \pm 0.28 \\
\hline
\end{tabular}
dunstig, und die Begleiter Io. bis I 2. Größe sind dann kaum Dresden besser gewesen sind, erscheint nach v. Engelhardts

Wenn man die im dritten Teile der Dresdener Beobachtungen niedergelegten Messungen berücksichtigt, ist jedes Paar von v. Engelhardt im Mittel $3.4 \mathrm{mal}$, von mir dagegen bisher 2 mal gemessen. Der w. F. einer Differenz würde somit in Mittel \pm 0 ." 88 betragen, was einem w. F. der Eigenbewegungen von \pm 0 ". I entspricht.

Aus den Messungen der physischen Paare habe ich indessen gefunden, daß $\mathrm{v}$. Engelhardt die Begleiter systematisch nördlicher setzt als ich, was einen scheinbaren "stardrift " gegen Süden verursachen würde. Aus 9 Paaren mit $30^{\prime \prime}$ bis $50^{\prime \prime}$ Distanz finde ich als systematischen Unterschied $\angle \delta=-0.16$, während 8 Paare mit 50" bis roo" Distanz $\angle \delta=-0.39$ ergeben. Da nur zwei physische Paare mit iiber 100" Distanz bei Engelhardt vorkommen, dürfte eine empirische Bestimmung dieser Fehler für die weiten Paare mit 100 " bis $300^{\prime \prime}$ Distanz kaum möglich sein, und ich habe daher versucht, die systematischen Fehler der abgeleiteten Eigenbewegungen bei der Ausgleichung zu bestimmen.

Die folgende Tafel enthält die Mittel der Messungen der optischen Paare korrigiert für Refraktion und bezogen auf das Aquinoktium I 900.0.

I. Messungen der Engelhardtschen Bradley-Sterne.

\begin{tabular}{|c|c|c|c|}
\hline \multirow[t]{2}{*}{ Br. $32 \times 2$} & I 892.34 & $248^{\circ} 5 \mathrm{I}^{\prime}$ & I $53^{\prime \prime} .96$ \\
\hline & 1908.43 & $250 \quad 34$ & 158.38 \\
\hline \multirow{2}{*}{ Br. 3212} & 1891.78 & 18258 & I $55.9^{6}$ \\
\hline & I 908.89 & I $S_{5}$ I I & I 53.28 \\
\hline \multirow[t]{2}{*}{$\alpha$ Andromedae } & 1887.08 & 27330 & 71.74 \\
\hline & 1908.07 & 27540 & 74.83 \\
\hline \multirow[t]{2}{*}{ Br. 90} & 1891.55 & 17136 & I 24.00 \\
\hline & 1908.65 & $1.7 \mathrm{I} \quad 14$ & I 26.89 \\
\hline \multirow[t]{2}{*}{47 Cassiopeiae } & I 887.26 & I96 40 & 94.68 \\
\hline & I 908.94 & I 98 I 6 & $95 \cdot 39$ \\
\hline \multirow[t]{2}{*}{ o Ceti } & I 888.06 & 8 I 20 & 116.27 \\
\hline & 1908.42 & 79 I 5 & II 6.94 \\
\hline \multirow[t]{2}{*}{ 2. Persei } & I 888.14 & 220 I 8 & 70.06 \\
\hline & I 9 IO. I I & 22539 & $74 \cdot 54$ \\
\hline \multirow[t]{2}{*}{ Br. $53^{6}$} & 1891.58 & $153 \quad 55$ & 95.08 \\
\hline & 1908.4 .3 & 15342 & $95 \cdot 7 \mathrm{I}$ \\
\hline \multirow[t]{2}{*}{$37 \mathrm{~A}^{1}$ Tauri } & 1887.08 & 188 I9 & $\mathrm{r} 37.4 \mathrm{r}$ \\
\hline & 1908.43 & I 89 I0 & I $37.2 \mathrm{I}$ \\
\hline \multirow[t]{2}{*}{$39 \mathrm{~A}^{2}$ Tauri } & I 887.08 & $35^{6}$ & $168.5^{\mathrm{I}}$ \\
\hline & 1908.43 & 241 & 169.59 \\
\hline
\end{tabular}

1). Publications of the Washburn Observatory. Vol. XII (I908).

2) Observations astronomiques faites par B. d'Engelhardt. Dresde. 1886-95. Die Messungen der Bradley-Sterne finden sich teils im zweiten Bande S. 34-80 teils im dritten Bande S. I I $^{-189}$. 


\begin{tabular}{|c|c|c|c|c|}
\hline 45 'Tauri & $\mathrm{I} 890.4 \mathrm{I}$ & $43^{\circ}$ & $15^{\prime}$ & $128^{\prime \prime} .36$ \\
\hline & 1908.99 & 42 & 16 & 125.67 \\
\hline $7 \pi t^{1}$ Orionis & 1891.09 & 248 & 6 & 172.19 \\
\hline & I 908.98 & 248 & 45 & $17 \pm .65$ \\
\hline I 3 Orionis & I 888.02 & 261 & I 5 & I 25.93 \\
\hline & 1909.07 & 264 & 42 & 124.14 \\
\hline$\alpha$ Aurigae & I 888.26 & 145 & 43 & 154.98 \\
\hline & 1908.93 & 144 & 29 & 146.40 \\
\hline$\lambda$ Aurigae & 1888.26 & 10 & 57 & 127.06 \\
\hline & I 908.93 & 5 & 31 & I 38.34 \\
\hline I I Tauri & I 888.08 & $27 \mathrm{I}$ & 24 & 77.11 \\
\hline & 1908.92 & 270 & 58 & 82.08 \\
\hline$\delta$ Aurigae & 1891.25 & 270 & I 6 & I I 3.84 \\
\hline & 1908.93 & $27 \mathrm{I}$ & 6 & 115.49 \\
\hline 71 Orionis & $\mathrm{I} 886.2 \mathrm{I}$ & 202 & 28 & 31.98 \\
\hline & 1909.16 & 202 & I 7 & 27.78 \\
\hline 7 Orionis & I 886.23 & 265 & 0 & 80.74 \\
\hline & 1909.16 & 254 & 2 & $87 \cdot 30$ \\
\hline $74 k$ Orionis & 1890.24 & 86 & 44 & 205.90 \\
\hline & 1909.06 & 87 & 53 & 203.99 \\
\hline $8 \mathrm{I}, y$ ncis & 1888.29 & $8 \mathrm{I}$ & $5^{2}$ & I 52.27 \\
\hline & 1909.04 & 79 & $5^{I}$ & I 57.05 \\
\hline $5^{6} \psi^{5}$ Aurigae & $1888.3^{2}$ & 22 & 25 & 46.63 \\
\hline & 1907.12 & 24 & 27 & 43.83 \\
\hline $5^{8} \psi^{7}$ Aurigae & 1891.27 & 106 & 40 & 40.93 \\
\hline & 1909.16 & 102 & 46 & 40.93 \\
\hline 20 Monocerotis & 1890.25 & 339 & 39 & 189.93 \\
\hline & 1909.07 & 3.39 & 27 & I 86.62 \\
\hline Br. 1048 & 1890.22 & 107 & 44 & 127.53 \\
\hline & I909.16 & 107 & 55 & 127.99 \\
\hline 22 I.yncis & 1890.27 & $3 \circ 3$ & 54 & 166.60 \\
\hline & I 909.16 & $3 \circ 3$ & 37 & I 68.94 \\
\hline$\varrho$ Geminorum & 1890.24 & 292 & 34 & 212.07 \\
\hline & 1909.16 & 291 & 17 & $2 \times 3.87$ \\
\hline 25 Monoccrotis & 1890.73 & 349 & 16 & 122.55 \\
\hline & 1909.16 & 349 & 44 & 121.66 \\
\hline 14 Canis min. & 1891.47 & 73 & 5.3 & 84.42 \\
\hline & 1909.07 & 75 & $3^{8}$ & 86.76 \\
\hline I 4 Canis min. & 1891.47 & $15 \mathrm{I}$ & 52 & I I 4.65 \\
\hline & 1909.07 & 150 & $5^{6}$ & I 17.82 \\
\hline o Ursac maj. & 1891.28 & $15^{2}$ & 22 & I 4.3 .88 \\
\hline & 1909.30 & I 5 I & 17 & 142.73 \\
\hline o Lisae maj. & 1891.20 & 208 & 6 & 176.59 \\
\hline & $1909 \cdot 30$ & 207 & $3^{I}$ & I 74.69 \\
\hline $22 \varphi^{1}$ Cancri & 1886.21 & 20 & $5^{6}$ & I 26.85 \\
\hline & 1909.26 & 20 & 30 & I 29.08 \\
\hline 39 Cancri & I 890.75 & $3 \circ 9$ & I 2 & I 34 . I 4 \\
\hline & $19 \circ 9 \cdot 31$ & $3 \circ 9$ & 4 & I 33.88 \\
\hline 39 Cancri & I 890.75 & I 10 & 33 & I 34.98 \\
\hline & 1909.25 & IIO & $4 I$ & I 34.45 \\
\hline$\gamma$ Cancri & 1890.75 & 65 & $5 I$ & 103.26 \\
\hline & I 900.26 & 65 & $5^{8}$ & 105.23 \\
\hline Io Ursae maj. & $\mathrm{r} 888.27$ & 202 & $3^{2}$ & I 36.47 \\
\hline & 1909.26 & 200 & I & I 29.47 \\
\hline 67 Cancri & I 888.32 & 324 & 19 & 101.47 \\
\hline & 1909.26 & 324 & 59 & 102.12 \\
\hline 75 Cancri & I $887.3^{8}$ & $4 I$ & I & 104.30 \\
\hline & 1909.26 & 39 & 18 & I I 1.57 \\
\hline Br. I 306 & $1890.3^{8}$ & 4 & I 3 & 147.32 \\
\hline & $1909 \cdot 3 \mathrm{I}$ & 4 & $4^{8}$ & I 44.39 \\
\hline
\end{tabular}

\begin{tabular}{|c|c|c|c|c|}
\hline \multirow[t]{2}{*}{40 Iyncis } & 1886.34 & $36^{\circ}$ & $7^{\prime}$ & 208.05 \\
\hline & 1909.26 & 37 & 2 & \\
\hline \multirow[t]{2}{*}{23 h Ursae maj. } & 1889.06 & 227 & 36 & 91.17 \\
\hline & 1909.31 & 227 & 49 & 93.72 \\
\hline \multirow[t]{2}{*}{4 I Lyncis } & 1888.30 & $16 \mathrm{I}$ & 44 & 81.21 \\
\hline & $1907 \cdot 32$ & $16 I$ & 30 & 79.59 \\
\hline \multirow[t]{2}{*}{4 I Lyncis } & 1888.30 & 80 & 39 & 83.58 \\
\hline & 1907.32 & 79 & 37 & $83 \cdot 52$ \\
\hline \multirow[t]{2}{*}{ o Leonis } & 1888.30 & $4 \mathrm{I}$ & 39 & 82.00 \\
\hline & 1909.26 & 42 & 26 & 83.86 \\
\hline \multirow[t]{2}{*}{$\varphi$ Leonis } & 1888.30 & 288 & 9 & 100.44 \\
\hline & 1908.74 & 288 & 28 & 98.39 \\
\hline \multirow[t]{2}{*}{62 Lrsac maj. } & 1888.33 & 298 & 59 & $9 \mathrm{r} .59$ \\
\hline & 1908.76 & 299 & 52 & $84 \cdot 3^{\circ}$ \\
\hline \multirow[t]{2}{*}{$\delta$ Trsae maj. } & 1890.50 & 73 & 15 & 191.76 \\
\hline & $1909 \cdot 3.3$ & 73 & 8 & 189.62 \\
\hline \multirow[t]{2}{*}{$\chi$ Virginis } & 1890.33 & I 37 & 44 & I 72.95 \\
\hline & 1908.74 & 137 & 39 & 172.96 \\
\hline \multirow[t]{2}{*}{ Virginis } & 1890.33 & 110 & 49 & 221.06 \\
\hline & I 908.74 & I IO & 40 & 222.53 \\
\hline \multirow[t]{2}{*}{$\chi$ Virginis } & 1890.33 & $33^{\circ}$ & 12 & 321.03 \\
\hline & I 908.74 & 330 & 35 & 321.39 \\
\hline \multirow[t]{2}{*}{33 Virginis } & $1887.3 \mathrm{I}$ & 87 & $3^{\circ}$ & 178.95 \\
\hline & I 908.25 & 190 & 42 & 171.55 \\
\hline \multirow[t]{2}{*}{ Br. 1820} & 1891.14 & 292 & 30 & Y 30.13 \\
\hline & I $909 \cdot 33$ & 293 & I 3 & 127.02 \\
\hline \multirow[t]{2}{*}{ Br. 1820} & 1891.14 & 215 & 44 & 159.07 \\
\hline & 1909.30 & $2 \times 4$ & 50 & 156.94 \\
\hline \multirow[t]{2}{*}{18 Bootis } & 1890.96 & 216 & 49 & I 54.68 \\
\hline & $19 \circ 9 \cdot 3 \mathrm{I}$ & 2 I 7 & 3 & I 56.44 \\
\hline \multirow[t]{2}{*}{18 Librac } & 1890.90 & 40 & 20 & $160.3^{2}$ \\
\hline & $1909 \cdot 36$ & 40 & I 2 & I $6.3 \cdot 38$ \\
\hline \multirow[t]{2}{*}{45 Bootis } & I 888.53 & 55 & 16 & 108.19 \\
\hline & $1909 \cdot 34$ & 52 & 55 & 107.37 \\
\hline 45 Bootis & 1889.04 & 49 & 21 & 251.67 \\
\hline & 1909.33 & 43 & 53 & $246.3^{2}$ \\
\hline - Coronae bor. & 1891.03 & $33^{6}$ & 42 & I 47.78 \\
\hline & 1909.29 & 337 & 45 & $146.8 \mathrm{I}$ \\
\hline$\psi$ Serpentis & 1890.96 & 279 & 50 & 172.17 \\
\hline & 1909.36 & 280 & 37 & I 72.28 \\
\hline I 5 Herculis & 1891.04 & 259 & I 5 & 65.97 \\
\hline & 1909.30 & 258 & 6 & $65.5^{6}$ \\
\hline$\sigma$ Coronae bor. & 1887.59 & 87 & 6 & 57.89 \\
\hline & 1909.47 & 86 & 9 & 63.97 \\
\hline 4 I Herculis & 1887.50 & 245 & 8 & 165.82 \\
\hline & $1909 \cdot 3^{2}$ & 246 & 23 & $158.9 \mathrm{I}$ \\
\hline Br. 2134 & $189 \mathrm{I} .0 \mathrm{I}$ & $2 \times 9$ & 35 & I 50.42 \\
\hline & 1909.38 & 219 & I I & $\mathrm{I} 50.6 \mathrm{I}$ \\
\hline 30 Ophiuchi & 1891.00 & 69 & 40 & 92.17 \\
\hline & I 909.38 & 69 & $3^{1}$ & 93.24 \\
\hline Br. 2 I 66 & I 89 I.I I & 266 & 44 & I 51.78 \\
\hline & $1909 \cdot 38$ & 267 & 33 & 152.30 \\
\hline $8_{3}$ Herculis & I 890.66 & 259 & 28 & I 59.72 \\
\hline & 1909.60 & 260 & I 8 & I 57.96 \\
\hline a Lyrac & I 886.75 & I 56 & 53 & 49.59 \\
\hline & 1905.46 & 163 & 29 & $53.5^{\circ}$ \\
\hline$\alpha$ Lyrae & 1886.76 & 39 & I I & I 30.66 \\
\hline & 1905.55 & 38 & 56 & I 24.52 \\
\hline IIEICun & I 886.76 & 247 & 8 & 96.22 \\
\hline & 1909.60 & 245 & 55 & 99.20 \\
\hline
\end{tabular}




\begin{tabular}{|c|c|c|c|c|}
\hline I I I Herculis & I 890.73 & $257^{\circ}$ & $33^{\prime}$ & I 19.16 \\
\hline & 1909.60 & 256 & 2.3 & I 2 I.32 \\
\hline I I I Herculis & 1890.73 & 264 & 33 & I 31.67 \\
\hline & I 909.60 & 263 & 48 & $133 \cdot 52$ \\
\hline I I Aquilae & $1886.7 \mathrm{r}$ & 261 & I I & I 6.66 \\
\hline & I908.26 & 269 & $\mathbf{I}$ & 17.08 \\
\hline$\varepsilon$ Aquilae & I 891.15 & I 87 & I 5 & 132.59 \\
\hline & I 909.63 & I 86 & 37 & I 3 I.I 4 \\
\hline$\varepsilon$ Aquilae & r 887.58 & 163 & 37 & 148.57 \\
\hline & I 909.63 & 163 & 6 & r 49.23 \\
\hline I 7 Lyrae & 1892.50 & 70 & 7 & 108.97 \\
\hline & r $9 \circ 9.68$ & 64 & 45 & I 33.49 \\
\hline I 7 Lyrae & 1891.29 & 296 & 9 & 124.84 \\
\hline & 1909.66 & 295 & 33 & 126.58 \\
\hline I 7 Lyrae & 1891.33 & I 16 & 9 & $15^{2.43}$ \\
\hline & 1909.68 & I 16 & 38 & 150.09 \\
\hline I 7 Lyrae & 1891.29 & 357 & 34 & 168.16 \\
\hline & 1909.66 & $35^{6}$ & 59 & 167.79 \\
\hline 3 I Aquilae & I 887.59 & $33^{2}$ & I 2 & I 17.09 \\
\hline & 1909.64 & $32 I$ & 42 & I 13.73 \\
\hline 3 I Aquilae & $\mathbf{r} 887.59$ & 353 & I 3 & I I 8.00 \\
\hline & r909.65 & 343 & 34 & 105.57 \\
\hline$\mu$ Aquilae & I 887.65 & 349 & $3^{8}$ & 59.60 \\
\hline & 1909.66 & 344 & I 9 & 64.20 \\
\hline$\mu$ Aquilae & I 890.68 & 71 & $3^{6}$ & $182.6 \mathrm{I}$ \\
\hline & I 908.59 & 70 & $3 \mathrm{I}$ & I 79.27 \\
\hline I 7 Cygni & $1887.8 \mathrm{I}$ & 142 & I & $148.8 \mathrm{I}$ \\
\hline & $1908.6 \mathrm{I}$ & 140 & 3 & $\mathrm{r} 4 \mathrm{r} \cdot 43$ \\
\hline$\alpha$ Aquilae & I 886.7 I & 309 & 56 & I 57.56 \\
\hline & 1905.60 & $3 \circ 5$ & 23 & $16 \times 85$ \\
\hline I 9 Cygni & I 89 I.3.3 & .102 & 30 & 54 . I I \\
\hline & 1908.90 & I04 & 28 & 53.72 \\
\hline I 5 Sagittae & 1886.78 & 272 & $2 \mathrm{I}$ & $20 \mathrm{I} .43$ \\
\hline & I 908.59 & 274 & 53 & I 93.66 \\
\hline$b^{3}$ Cygni & 1890.16 & 23 & 17 & $225.1 \mathrm{~T}$ \\
\hline & 1908.66 & 23 & 8 & 222.22 \\
\hline I 5 Delphini & I 890.65 & $8 r$ & I 4 & 109.00 \\
\hline & r 908.59 & 82 & 32 & IO8. I I \\
\hline I8 Delphini & I 890.37 & 162 & 28 & $197.3^{6}$ \\
\hline & I907.68 & I 62 & 3 & I 97.24 \\
\hline$\delta$ Equulei & 1886.87 & $2 \mathrm{I}$ & 25 & 40.25 \\
\hline & 1908.59 & I 7 & r 5 & 45.87 \\
\hline
\end{tabular}

\begin{tabular}{|c|c|c|c|c|}
\hline Br. 2926 & $\mathrm{I} 89 \mathrm{I} .4 \mathrm{I}$ & $36^{\circ}$ & $8^{\prime}$ & $79^{\prime \prime} 59$ \\
\hline & 1908.72 & 34 & 26 & 75.02 \\
\hline $\boldsymbol{\varepsilon}$ Cephei & I $89 \mathrm{I} .4 \mathrm{I}$ & 19 & 54 & I 32.65 \\
\hline & 1908.72 & 17 & 19 & I 29. I 5 \\
\hline 35 Pegasi & 1 891.59 & 208 & 29 & 101.51 \\
\hline & 1908.72 & 210 & 28 & $98 \cdot 3^{I}$ \\
\hline 35 Pegasi & I 892.13 & 240 & I 3 & 181.79 \\
\hline & 1908.72 & $24 \mathrm{I}$ & $3^{6}$ & I 8 I. 54 \\
\hline Br. 3077 & $1887.9 \mathrm{I}$ & 183 & $5 I$ & $88.3^{6}$ \\
\hline & 1908.72 & 207 & 47 & 106.57 \\
\hline Br. 3094 & I 891.42 & $3 \circ 3$ & I 5 & $8 \mathrm{I} .25$ \\
\hline & I 908.72 & 301 & 7 & 84.10 \\
\hline I 2 Andromed. & I 890.95 & 255 & 40 & I I 9.02 \\
\hline & I908.73 & 256 & 5 & 120.54 \\
\hline Br. 3107 & I 89 I.36 & 322 & 33 & 75.09 \\
\hline & 1908.72 & 319 & $2 I$ & $77.7 \mathrm{I}$ \\
\hline$x$ Piscium & 1891.36 & 344 & 45 & I 59.87 \\
\hline & r 908.72 & 344 & I 7 & 161.77 \\
\hline 74 Pegasi & 1890.96 & 35 & 47 & 99.92 \\
\hline & I 908.87 & 34 & 26 & $97 \cdot 74$ \\
\hline 85 Pegasi & I 886.99 & $3^{66}$ & 7 & 2 I.I 5 \\
\hline & 1908.90 & $33^{8}$ & I 8 & $45 \cdot 47$ \\
\hline
\end{tabular}

Ein Vergleich der Größenschätzungen mit den HarvardMessungen ${ }^{1)}$ von 35 Sternen gibt folgende Beziehungen:

\begin{tabular}{|c|c|c|c|c|}
\hline $\begin{array}{l}\text { Lau } \\
8^{\mathrm{m}} \cdot 77\end{array}$ & $=$ & $\begin{array}{c}\text { Harvard } \\
9^{\mathrm{m}} \cdot \mathrm{O} 2\end{array}$ & $\begin{array}{l}\text { Engelhardt } \\
8^{\mathrm{m}} \cdot 39=\end{array}$ & $\begin{array}{c}\text { Harvaro } \\
8^{m} \cdot 49\end{array}$ \\
\hline $9.4 \mathrm{I}$ & $=$ & $9 \cdot 53$ & $9 \cdot 32=$ & 9.47 \\
\hline I 0.04 & $=$ & 9.80 & $9.89=$ & 9.98 \\
\hline 10.59 & $=$ & 10.25 & 10.60 & 10.37 \\
\hline
\end{tabular}

Die Ergebnisse der Beobachtungen der optischen Paare sind in der folgenden Tabelle zusammengestellt. Es enthält die erste Spalte die Bezeichnung des Hauptsternes; die zweite die Harvard-Größe des Begleiters nach v. Engelhardts und meinen eigenen Schätzungen; die dritte und vierte die jährliche relative Bewegung in $\alpha \cdot \cos \delta$ und $\delta$; die fünte und sechste die absoluten Eigenbewegungen des Hauptsternes; die beiden letzten Spalten die entsprechenden Größen für den Begleiter. Die Angaben über die Eigenbewegung der Hauptsterne verdanke ich einer brieflichen Mitteilung des Herrn Prof. Boss.

II. Eigenbewegungen der Engelhardtschen Bradley-Sterne.

\begin{tabular}{|c|c|c|c|c|c|c|c|}
\hline Name & Größe & \multicolumn{2}{|c|}{ Jährl. rel. Bewegung } & \multicolumn{2}{|c|}{ E. B. d. Hauptsternes } & \multicolumn{2}{|c|}{ E.B. d. Begleiters } \\
\hline Br. 32 I 2 & $9^{m} \cdot 7$ & -0.359 & +o." I 78 & +0.380 & -0.1176 & $+0.02 \mathrm{I}$ & +0.002 \\
\hline$\gg$ & 10.6 & -0.337 & +0.181 & +0.380 & -0.176 & +0.043 & +0.005 \\
\hline $\boldsymbol{\alpha}$ Andromedae & 9.9 & -0.136 & +0.143 & +0.140 & $-0.16 \mathrm{I}$ & +0.004 & -0.018 \\
\hline Br. 90 & 9.2 & +0.072 & -0.160 & -0.072 & +0.174 & 0.000 & +0.014 \\
\hline$\vartheta$ Persei & 10.7 & -0.359 & +0.060 & +0.339 & -0.090 & -0.020 & -0.030 \\
\hline 47 Cassiopeiae & $9 \cdot 7$ & -0.127 & +0.006 & +0.133 & -0.054 & +0.006 & -0.048 \\
\hline o Ceti & 9.6 & -0.004 & +0.212 & $-0.00 \mathrm{I}$ & -0.237 & -0.005 & -0.025 \\
\hline Br. 536 & 9.4 & +0.036 & -0.024 & -0.023 & -0.013 & +0.013 & -0.037 \\
\hline $37 \mathrm{~A}^{1}$ Tauri & $9 \cdot 4$ & -0.093 & +0.024 & +0.093 & -0.064 & 0.000 & -0.040 \\
\hline $39 \mathrm{~A}^{2}$ Tauri & 8.2 & -0.186 & +0.060 & +0.174 & -0.138 & -0.012 & -0.078 \\
\hline 5 Tauri & 10.5 & -0.184 & -0.026 & +0.142 & +0.013 & -0.042 & -0.013 \\
\hline Orionis & 9.1 & -0.012 & +0.112 & +0.055 & -0.134 & +0.043 & -0.022 \\
\hline
\end{tabular}

1) Annals of Harvard College Observatory. Vol. 64 No. VI. 


\begin{tabular}{|c|c|c|c|c|c|c|c|}
\hline Name & Größe & Jährl. rel. & Bewegung & E. B. d. $\mathrm{H}$ & uptsternes & E. B. d. & Begleiters \\
\hline I. 3 Orionis & $\mathrm{I} \mathrm{O}^{\mathrm{m}} \mathrm{I}$ & $+0.0_{4} \mathrm{I}$ & $+0.3^{6} 5$ & +o."006 & $-0.38 \mathrm{I}$ & +0.047 & - -."0 I 6 \\
\hline$\alpha$ Aurigae & 9.8 & -0.109 & +0.430 & +0.086 & -0.429 & -0.023 & +0.001 \\
\hline$\lambda$ Aurigae & $9 \cdot 7$ & -0.524 & +0.627 & $+0.53^{\circ}$ & -0.656 & +0.006 & -0.029 \\
\hline I I I Tauri & $9 \cdot 4$ & -0.239 & -0.024 & +0.245 & -0.010 & +0.006 & -0.034 \\
\hline$\delta$ Aurigae & 9.8 & --0.092 & +0.096 & +0.086 & -0.126 & -0.006 & -0.030 \\
\hline 7 I Orionis & 10.6 & +0.074 & +0.168 & -0.098 & $-0.20 \mathrm{I}$ & -0.024 & -0.033 \\
\hline$"$ & I I. 2 & -o.1 51 & -0.744 & -0.098 & $-0.20 \mathrm{I}$ & -0.249 & $-0.945^{*}$ \\
\hline $74 k$ Orionis & 9.4 & $-0.09 \mathrm{I}$ & -0.223 & +0.088 & +0.193 & -0.003 & -0.030 \\
\hline 8 Lyncis & 9.0 & +0.186 & +0.296 & -0.194 & $-0.28 \mathrm{I}$ & -0.008 & +0.015 \\
\hline $5^{6} \psi^{5}$ Aurigae & 8.4 & +0.019 & $-0.17 \mathrm{I}$ & +0.008 & $+0.15^{8}$ & +0.027 & -0.013 \\
\hline $5^{8} \psi^{7}$ Aurigae & IO. I & +0.040 & +0.151 & -0.018 & -0.135 & +0.022 & +0.016 \\
\hline 20 Monocerotis & 9.6 & +0.029 & -0.177 & 0.000 & +0.217 & +0.029 & +0.040 \\
\hline Br. 1048 & 10.5 & +0.028 & -0.016 & -0.001 & -0.016 & +0.027 & -0.032 \\
\hline 22 Iyncis & 9.8 & -0.128 & +0.032 & 十0.11 8 & -0.085 & -0.010 & -0.053 \\
\hline @ Geminorum & 10.5 & -0.182 & -0.198 & +0.149 & +0.183 & -0.033 & -0.015 \\
\hline 25 Monocerotis & 10.5 & +0.062 & -0.038 & -0.070 & +0.018 & -0.008 & --0.020 \\
\hline I 4 Canis min. & $9 \cdot 3$ & +0.167 & -0.108 & -o.159 & +0.095 & +0.008 & $-0.0 \mathrm{I} 3$ \\
\hline  & 9.4 & +0.181 & -0.104 & -0.159 & +0.095 & +0.022 & -0.009 \\
\hline o Ursae maj. & 10.2 & +0.103 & +0.127 & $-0.12 \mathrm{I}$ & -0.114 & -0.018 & +0.013 \\
\hline 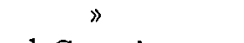 & 10.4 & +0.136 & +0.047 & $-0.12 I$ & - - .114 & +0.015 & -0.067 \\
\hline$\varphi^{1}$ Cancri & 10.4 & -0.005 & +0.105 & -0.030 & $-0.13 I$ & -0.035 & -0.026 \\
\hline 39 Cancri & 9.2 & 0.000 & -0.022 & -0.038 & -0.022 & -0.038 & -0.044 \\
\hline$"$ & $9 \cdot 3$ & -0.033 & 0.000 & $-0.03^{8}$ & -0.022 & -0.071 & -0.022 \\
\hline$\gamma$ Cancri & $9 \cdot 7$ & +0.106 & +0.024 & -0.102 & $-0.05^{\circ}$ & +0.004 & -0.026 \\
\hline Io Ursae maj. & $9 \cdot 9$ & $+0.3^{80}$ & +0.210 & $-0.4 \dot{3}^{1}$ & $-0.26 \mathrm{I}$ & $-0.05 \mathrm{I}$ & $-0.05 \mathrm{I}$ \\
\hline 67 Cancri & 9.1 & +0.028 & $+0.05^{8}$ & $\cdots 0.05^{8}$ & -0.088 & -0.030 & -0.030 \\
\hline 75 Cancri & $9 \cdot 5$ & +0.101 & +0.349 & -0.116 & $-0.3^{87}$ & -0.015 & -0.038 \\
\hline Br. I 306 & 9.8 & +0.066 & - o.I 6 I & $-0.03^{8}$ & +o.1 35 & +0.028 & -0.026 \\
\hline 40 Lyncis & $9 \cdot 5$ & +0.189 & +0.010 & -0.217 & +0.010 & -0.028 & +0.020 \\
\hline$h$ Ursae maj. & 10.8 & -0.105 & -0.072 & +0.114 & +0.025 & +0.009 & -0.047 \\
\hline 4I Lyncis & 8.5 & -0.010 & +0.086 & -0.003 & -0.136 & -0.013 & $-0.05^{\circ}$ \\
\hline$»$ & IO.I & -0.017 & +0.077 & -0.003 & $-0.13^{6}$ & -0.020 & -0.059 \\
\hline o Leonis & 10.7 & +0.100 & +0.030 & - 0.145 & -0.039 & -0.045 & -0.009 \\
\hline$\varphi$ Leonis & $9 \cdot 3$ & +0.104 & -0.006 & -O.II 2 & -0.044 & -0.008 & -0.050 \\
\hline 62 Ursae maj. & $9 \cdot 3$ & +0.341 & $-0.12 \mathrm{I}$ & -0.346 & +0.011 & -0.005 & -0.110 \\
\hline$\delta$ Ursae maj. & 10.0 & $-0.1 \mathrm{I} 5$ & -0.013 & +O.I Io & +0.003 & -0.005 & -0.010 \\
\hline$\chi$ Virginis & 9.2 & +0.010 & +0.008 & -0.076 & -0.037 & -0.066 & -0.029 \\
\hline$"$ & 10.3 & +0.055 & +0.006 & -0.076 & -0.037 & $-0.02 \mathrm{I}$ & $-0.03 I$ \\
\hline$"$ & 9.0 & +0.038 & +0.074 & -0.076 & -0.037 & $-0.03^{8}$ & +0.037 \\
\hline 33 Virginis & 9.1 & -0.405 & +0.423 & +0.272 & $-0.45^{6}$ & -0.133 & -0.033 \\
\hline $\mathrm{Br} .1820$ & I 0.7 & +0.192 & +0.015 & -0.164 & -0.030 & +0.028 & 一.0.01 5 \\
\hline$\gg$ & 10.9 & +0.179 & -0.016 & -0.164 & -0.030 & +0.015 & -0.046 \\
\hline I 8 Bootis & 9.8 & -0.074 & -0.056 & +0.108 & -0.038 & +0.034 & -0.094 \\
\hline I 8 Librae & I 0.4 & +0.092 & +0.140 & -0.106 & -0.076 & -0.014 & +0.064 \\
\hline 45 Bootis & I 0.8 & -o.1 55 & +0.148 & +0.186 & -0.183 & +0.031 & -0.035 \\
\hline$”$ & $9 \cdot 7$ & -0.995 & +0.670 & +0.186 & -0.183 & -0.809 & $+0.487 *$ \\
\hline o Coronae bor. & $9 \cdot 7$ & +o.1 57 & +0.008 & -0.125 & -0.058 & +0.032 & $-0.05^{\circ}$ \\
\hline$\psi$ Serpentis & 10.5 & +0.017 & +0.127 & -0.085 & -0.150 & -0.068 & -0.023 \\
\hline I 5 Herculis & 9.8 & +0.036 & -0.066 & $-0.05^{\circ}$ & +0.030 & -0.014 & -0.036 \\
\hline$\sigma$ Coronae bor. & 10.0 & +0.274 & +0.068 & $-0.27 \mathrm{I}$ & -0.090 & +0.003 & -0.022 \\
\hline 4 I Herculis & 9.6 & +0.222 & +0.278 & -0.218 & --0.277 & +0.004 & +0.001 \\
\hline Br. 2134 & 9.1 & +0.038 & -0.044 & $-0.03 I$ & -0.019 & +0.007 & -0.063 \\
\hline 3. Ophiuchi & 9.9 & $+0.05^{\circ}$ & +0.033 & -0.054 & -0.088 & -0.004 & -0.055 \\
\hline Br. 2 r 66 & 9.8 & -0.035 & +0.117 & -0.015 & -0.134 & -0.050 & -0.017 \\
\hline $8_{3}$ Herculis & $9 \cdot 5$ & +0.070 & +0.137 & -0.063 & -0.115 & +0.007 & +0.022 \\
\hline$\alpha$ Lyrae & 9.0 & -0.174 & $-0.3 \circ 5$ & +0.204 & +0.279 & +0.030 & -0.026 \\
\hline 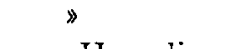 & IO.I & -0.229 & -0.235 & +0.204 & +0.279 & -0.025 & +0.044 \\
\hline Hercuhs & 10.7 & -0.084 & -0 & $+0.06_{3}$ & +0.106 & $02 \mathrm{I}$ & -0.029 \\
\hline
\end{tabular}




\begin{tabular}{|c|c|c|c|}
\hline Name & Größe & \multicolumn{2}{|c|}{ Jährl. rel. Bewegung } \\
\hline I I Herculis & $9^{11} \cdot 9$ & --0.082 & 一 - 152 \\
\hline$»$ & 10.4 & -0.088 & $-0.10 \mathrm{I}$ \\
\hline 1 I Aquilae & $9 \cdot 7$ & -0.028 & $+0.10_{4}$ \\
\hline$\varepsilon$ Aquilae & 10.3 & +0.100 & +0.067 \\
\hline$»$ & 10.7 & +0.067 & +0.011 \\
\hline I 7 Lyrae & I 0.8 & +1.057 & $+\mathrm{I} . \mathrm{I} 7 \mathrm{I}$ \\
\hline$»$ & 9.2 & -0.123 & -0.036 \\
\hline » & 10.2 & $-0.13^{8}$ & +0.009 \\
\hline$»$ & 9.8 & - 0.1 II & -0.025 \\
\hline 3 I Aquilae & IO.I & -0.720 & -0.659 \\
\hline$》$ & $9 \cdot 3$ & -0.732 & -0.724 \\
\hline$\mu$ Aquilae & I I. 5 & $-0.30 \mathrm{r}$ & +0.144 \\
\hline 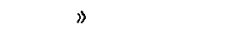 & $9 \cdot 5$ & $-0.23^{8}$ & +0.120 \\
\hline 7 Cygni & 9.2 & --0.037 & +0.426 \\
\hline$\alpha$ Aquilae & $9 \cdot 5$ & $-0.59^{\circ}$ & -0.393 \\
\hline I9 Cygni & 10.6 & -0.046 & -0.097 \\
\hline I 5 Sagittae & 9.1 & $+0.38 I$ & +0.377 \\
\hline$b^{3}$ Cygni & $9 \cdot 5$ & $-0.09 \mathrm{I}$ & -0.131 \\
\hline I 5 Delphini & 10.4 & -0.030 & -0.143 \\
\hline I 8 Delphini & 10.1 & +0.077 & $+0.03 \mathrm{I}$ \\
\hline$\delta$ Equatiei & 10.4 & $-0.05 \mathrm{I}$ & +0.292 \\
\hline Br. 2926 & IO. I & -0.260 & -0.139 \\
\hline$\varepsilon$ Cephei & $9 \cdot 5$ & -0.388 & -0.083 \\
\hline 35 Pegasi & 10.2 & -0.084 & +0.262 \\
\hline$»$ & IO. I & -0.110 & +0.225 \\
\hline Br. 3077 & 9.6 & -2.102 & -0.294 \\
\hline Br. 3094 & 10.0 & -0.234 & -0.063 \\
\hline I 2 Andromed. & $9 \cdot 4$ & -0.094 & +0.027 \\
\hline Br. 3107 & 10.5 & -0.286 & -0.037 \\
\hline * Piscium & 10.3 & -0.102 & +0.085 \\
\hline 74 Pegasi & 10.4 & -0.176 & -0.025 \\
\hline 85 Pegasi & 9.5 & -0.870 & +0.968 \\
\hline
\end{tabular}

Unter den Eigenbewegungen der Begleiter sind negative Werte auffallend häufig. Nach Ausschluß der mit * bezeichneten Objekte mit anomalen Eigenbewegungen findet man im Mittel $\mu_{\alpha}=-0$. .о 10 $\mu_{\delta}=-0.025$.

Nach den Ergebnissen der Messungen der physischen Paare glaube ich die Ursache dieses scheinbaren "star-drift « in den systematischen Fehlern der Mikrometermessungen suchen zu müssen. I)iese Annahme wird durch die Tatsache gestützt, daß die scheinbare »drift" in Deklination sowohl von der Helligkeit $m_{\mathrm{B}}$ des Begleiters als auch von der Deklination $\delta$ unabhängig ist, wie aus dem folgenden Täfelchen ersichtlich ist:

\begin{tabular}{rcc|ccc}
$m_{\mathrm{B}}$ & $\mu_{J}$ & $n$ & $\delta$ & $\mu_{J^{\prime}}$ & $n$ \\
$8^{\mathrm{m}} 8$ & -0.029 & I 3 & -5.8 & $-0.0 \mathrm{I} 3$ & I 3 \\
$9 . \mathrm{I}$ & -0.024 & I 2 & +5.0 & -0.025 & I 3 \\
9.4 & -0.032 & 13 & $+\mathrm{I} 4.1$ & -0.035 & 22 \\
9.6 & -0.025 & I 2 & +25.1 & -0.025 & I 5 \\
9.9 & -0.014 & I 2 & +34.6 & -0.022 & I4 \\
10.2 & -0.020 & I 2 & +45.2 & -0.030 & I0 \\
10.4 & -0.026 & I 3 & +55.2 & -0.019 & 6 \\
10.9 & -0.038 & 1 2 & +64.1 & -0.020 & 6
\end{tabular}

Die gemeinsame Bewegung der Begleiter dürfte vielmehr von der verschiedenen Auffassung der Mitte der Scheibchen

$$
\begin{array}{llll}
\text { E.B.d. Hauptsternes } & \multicolumn{2}{c}{\text { E.B. d. Begleiters }} \\
+0.063 & +0.106 & -0.019 & -0.046 \\
+0.063 & +0.106 & -0.025 & +0.005 \\
+0.001 & -0.120 & --0.027 & -0.016 \\
-0.064 & -0.077 & +0.036 & -0.010 \\
-0.064 & -0.077 & +0.003 & -0.066 \\
+-0.120 & +0.013 & +1.177 & +1.184 \\
+0.120 & +0.013 & -0.003 & -0.023 \\
+0.120 & +0.013 & -0.018 & +0.022 \\
+0.120 & +0.013 & +0.009 & -0.012 \\
+0.725 & +0.630 & +0.005 & -0.029 \\
+0.725 & +0.630 & -0.007 & -0.094 \\
+0.213 & -0.152 & -0.088 & -0.008 \\
+0.213 & -0.152 & -0.025 & -0.032 \\
+0.011 & -0.452 & -0.026 & -0.026 \\
+0.535 & +0.380 & -0.055 & -0.013 \\
+0.011 & +0.097 & -0.035 & 0.000 \\
-0.404 & -0.413 & -0.023 & -0.036 \\
+0.065 & +0.068 & -0.026 & -0.063 \\
+0.062 & +0.096 & +0.032 & -0.047 \\
+-0.065 & -0.053 & +0.012 & -0.022 \\
+0.041 & -0.303 & -0.010 & -0.011 \\
+0.239 & +0.127 & -0.021 & -0.012 \\
+0.450 & +0.044 & +0.062 & -0.039 \\
+0.076 & -0.316 & -0.008 & -0.054 \\
+0.076 & -0.316 & -0.034 & -0.091 \\
+0.082 & +0.296 & -0.020 & +0.002 \\
+0.204 & +0.031 & -0.030 & -0.032 \\
+0.115 & -0.078 & +0.021 & -0.051 \\
+0.318 & -0.019 & +0.032 & -0.056 \\
+0.085 & -0.090 & -0.017 & -0.005 \\
+0.120 & -0.002 & -0.056 & -0.027 \\
+0.835 & -0.986 & -0.035 & -0.018
\end{array}
$$

der hellen Hauptsterne herriihren. Ich finde nämlich, daß die scheinbaren Eigenbewegungen von dem Positionswinkel $p$ des Begleiters unabhängig sind:

$$
\begin{aligned}
& \begin{array}{llll|llll}
p & \mu_{r s} \cos \delta^{\prime} & \mu_{j} & n & p & \mu_{u} \cos \delta^{\prime} & \mu_{\delta} & n
\end{array}
\end{aligned}
$$

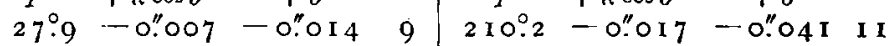

$$
\begin{aligned}
& \begin{array}{llll|llll}
57.9 & -0.011 & -0.017 & 10 & 256.3 & -0.008 & -0.025 & 12
\end{array} \\
& \begin{array}{llll|lllll}
88.3 & -0.002 & -0.019 & 8 & 283.9 & -0.011 & -0.036 & 10
\end{array} \\
& \begin{array}{llll|lllll} 
& \text { I } 30.3 & -0.022 & -0.013 & 9 & 3 \text { I } 5.8 & -0.020 & -0.028 & 8
\end{array} \\
& \begin{array}{lllll|llll}
174.0 & 0.000 & -0.024 & \text { I I } & 35 \text { I.9 } & -0.006 & -0.027 & \text { I I }
\end{array}
\end{aligned}
$$

Bei der Ausgleichung habe ich daher in den Bedingungsgleichungen außer der scheinbaren Sonnenbewegung $\mu$ und dem Korrektionsfaktor $\left(1+\frac{\eta^{\prime \prime}}{50^{\prime \prime}}\right)$ der Newcombschen Präzessionskonstante noch die konstanten Fehler $z_{c c}$ und $z_{\jmath}$ der abgeleiteten Eigenbewegungen aufgenommen.

$$
\text { Die Koeffizienten } \frac{\mathrm{d}(\alpha \cos \delta)}{\mathrm{d} \eta} \text { und } \frac{\mathrm{d} \delta}{\mathrm{d} \eta} \text { ergeben sich aus }
$$

den Bossschen Präzessionen in $\alpha$ und $\delta$. Bei der Berechnung der Koeffizienten von $\mu$ habe ich auf die verschiedenen Heiligkeiten der Sterne keine Rücksicht genommen. Bei einer völlig strengen Ausgleichung müßte man nämlich eher auf die (unbekannten) Spektraltypen der Begleiter Rücksicht nehmen als auf die nur bis $\pm \mathrm{I}^{\mathrm{m}} \cdot 5$ reichenden Helligkeitsunterschiede. ${ }^{1}$ ) 
Da die Koeffizienten der Bedingungsgleichungen sich mittels der Kapteynschen Werte ${ }^{1}$ ) von $\chi$ und $\lambda$ leicht herstellen lassen, unterlasse ich es, die 198 Gleichungen hier anzuführen. Um bequeme Arbeitszahlen zu erhalten, habe ich vor der Ausgleichung vorläufige Korrektionen von +o."O ro bezw. +0.025 an die Eigenbewegungen angebracht.

Die partiellen Normalgleichungen sind:

Aus den Gleichungen in $\mu_{6 s} \cos \delta$

$$
\begin{aligned}
& +99.00 z_{6 \ell}-0.28 \mu+79.87 \eta=-0.0070 \\
& -0.28+28.56-2.37=+0.1183 \\
& +79.87-2.37+68.50=-0.0620
\end{aligned}
$$

Aus den Gleichungen in $\mu_{\sigma}$

$$
\begin{aligned}
& +99.00 z_{\delta}-46.09 \mu+0.07 \eta=-0.0440 \\
& -46.09+30.19+2.49=+0.0546 \\
& +0.07+2.49+6.6 \mathrm{r}=+0.0179
\end{aligned}
$$

Die Auflösung gibt:

$$
\begin{array}{ll}
\eta=-0.00347 & z_{\jmath}=+0.0015 \\
\mu=+0.00412 & z_{f t}=+0.0028
\end{array}
$$

Bei der Ausgleichung habe ich allen Gleichungen das Gewicht I beigelegt. Dieses Verfahren wird durch die Verteilung der Restfehler gerechtfertigt. Als w. F. einer Gleichung findet man nämlich für verschiedene Helligkeiten der Begleiter:

$$
\begin{array}{ccc}
\text { Helligkeit } & \alpha \cos \delta & \delta \\
8^{\mathrm{m}} \cdot 5^{-} 9^{\mathrm{m}} \cdot 5 & \pm 0.024 & \pm 0.019 \\
9.5-10.5 & \pm 0.016 & \pm 0.020 \\
\text { I0.5-II.5 } & \pm 0.020 & \pm 0.019
\end{array}
$$

Eine Beziehung zwischen den Restfehlern und der galaktischen Breite $\beta$ tritt nicht hervor. Die w. F. für verschiedene Breiten sind:

$$
\begin{array}{rccc}
\text { Gal. Breite } & \beta_{m} & \alpha \cos 8 & \delta \\
60^{\circ}-30^{\circ} \mathrm{S} & -41.6 & \pm 0.017 & \pm 0.019 \\
30-0 \mathrm{~S} & -\mathrm{I} 1.0 & \pm 0.022 & \pm 0.017 \\
0-30 \mathrm{~N} & +\mathrm{I} 3.7 & \pm 0.015 & \pm 0.019 \\
30-60 . \mathrm{N} & +44.6 & \pm 0.020 & \pm 0.019
\end{array}
$$

Bei der Ausgleichung habe ich drei Sterne mit anomalen Eigenbewegungen ausgeschlossen. Eine partielle Ausgleichung gab nämlich für diese Sterne $\mu=+1$ "32, während die 99 übrigen $\mu=+0.004$ gaben. Diese drei Sterne bilden somit etwas für sich. Da ihre absolute Helligkeit kaum ein Hunderttausendstel der Helligkeit der übrigen Sterne beträgt,

Urania-Sternwarte, April I 9 Io. stellen sie vielleicht die letzte Stufe ("schwarze Sonnen") der spektralen Entwickelung dar.

Die Korrektion der Newcombschen Präzessionskonstante $\eta=-0.0035$ stimmt befriedigend mit der von Comstock gefundenen überein. Die Präzessionskonstante für I 850.0 wäre hiernach $50^{\prime \prime} 24 \mathrm{I} 8$.

Das Hauptinteresse knüpft sich jedoch an den Wert von $\mu$. Mit Campbells Geschwindigkeit der Sonne $(4.2$ Erdbahnhalbmesser) finden wir aus $\mu=+0.004$ r 2 als mittlere Parallaxe der Sterne Io. Größe $\left.{ }^{2}\right)$ :

$$
\pi_{10}=0 \text {."0010. }
$$

Dieser Wert ist erheblich kleiner als der von Comstock gefundene. Zugunsten meiner Bestimmung möchte ich hervorheben, daß die systematischen Fehler soweit als möglich berücksichtigt sind, was bei Untersuchungen dieser Art eine unabweisbare Forderung sein muß. Aus den rohen Eigenbewegungen der Engelhardt-Sterne würde man wegen der systematischen Fehler der $\mu_{\delta}$ als Säkularbewegung der Sonne $3^{\prime \prime}$ statt $0^{\prime \prime} 4$ erhalten; man hätte somit aus meinen Daten selbst bei einem Zeitintervall von einem halben Jahrhundert bei Vernachlässigung der systematischen Fehler ein mit Comstocks übereinstimmendes Resultat herausbringen können.

Die vorliegende Untersuchung ergibt in Übereinstimmung mit Comstock, daß die Eigenbewegungen der lichtschwachen Sterne größer sind, als man nach ihrer Helligkeit bezw. Parallaxe erwarten sollte. Die Tatsache, daß die systematischen Fehler der Messungen scheinbare Bewegungen von über $2^{\prime \prime}$ im Jahrhundert bewirken können, muß jedoch gegen ein allzugroßes Vertrauen in die Ergebnisse der Mikrometermessungen warnen.

Nach den bisherigen Untersuchungen scheinen die Sterne

\begin{tabular}{|c|c|c|c|c|c|c|c|c|c|c|c|}
\hline I 910 & szeit & $\Delta \alpha$ & $\lambda \delta$ & Vgl. & Bb. & & $|\log p \cdot \Lambda|$ & & & $\operatorname{Re}$ & * \\
\hline \multicolumn{12}{|c|}{ Am ro.Zöller der Sternwarte Leiden von Observator 7 . Voûte. (Fortsetzung von A. N. 4389.) } \\
\hline &  & $\left|\begin{array}{rr}-0^{m} & 1 \\
-1 & 7.70 \\
-1 & 7.65\end{array}\right|$ & $\begin{array}{l}-0^{\prime} \text { I } 8.6 \\
+456.9\end{array}$ & & $\begin{array}{l}\text { V } \\
\text { V }\end{array}$ & $\begin{array}{lll}2 \mathrm{I}^{\mathrm{h}} & 44^{\mathrm{m}} \mathrm{I} 0^{5} .56 \\
2 \mathrm{I} & 44 & 10.77\end{array}$ & & & & $\begin{array}{l}-\mathrm{r} .85-1 \\
-\mathrm{r} .85-\mathrm{r}\end{array}$ & \\
\hline 9 & 6 I 6 & to 47.47 & $+44 \mathrm{I} .4$ & I 2,6 & $\mathrm{~V}$ & $\begin{array}{lll}21 & 52 & 35.75\end{array}$ & 9.558 & +7 1 2 & 0.832 & $-1.82-10.9$ & \\
\hline
\end{tabular}
des Typus I besonders häufig in der Milchstraße zu sein. Es dürfte daher vielleicht mehr als ein Zufall sein, daß die gefundene mittlere Parallaxe der Sterne Io. Größe mit der Annahme stimmt, daß die lichtschwachen Sterne dem Typus I angehören. Aus Kapteyns Angaben ${ }^{3}$ ) findet man mit Campbells Sonnengeschwindigkeit die mittlere Parallaxe der Sterne des Typus I für die Größe $5^{\mathrm{m}} \cdot 5$ gleich $0 . " 0086$; die Parallaxe der Sterne I0. Größe wäre somit $\pi_{10}=0$ ".00г I. Aus den gemessenen Parallaxen der A-Sterne findet Hertzsprung (l.c.) $\pi_{0}=0$." 53 oder $\pi_{10}=0$. .0015. Wir sind daher vorläufig nicht genötigt, eine besondere Konstitution der lichtschwachen Sterne oder eine merkbare Absorption im Weltenraume anzunehmen.

H. E. Lau.

1) Publications of the Astronomical Laboratory at Groningen no. 9 (I9O2).

2) Die mittlere Helligkeit der 99 Sterne ist 9.84 Harvard oder Io.o I Potsdam.

3) Astronomische Nachrichten Band 146, S. I Io.

Beobachtungen des Kometen 1910 a. 